\title{
Optimal Control of Natural Eye-Head Movements Minimizes the Impact of Noise
}

\author{
Murat Sağlam, ${ }^{1,4}$ Nadine Lehnen, ${ }^{2,3}$ and Stefan Glasauer ${ }^{1,3,4}$ \\ ${ }^{1}$ Institute for Clinical Neurosciences, ${ }^{2}$ Department of Neurology, ${ }^{3}$ Integrated Center for Research and Treatment of Vertigo, Balance and Ocular Motor \\ Disorders, and ${ }^{4}$ Bernstein Center for Computational Neuroscience, Ludwig-Maximilians-University, 81377 Munich, Germany
}

When shifting gaze to foveate a new target, humans mostly choose a unique set of eye and head movements from an infinite number of possible combinations. This stereotypy suggests that a general principle governs the movement choice. Here, we show that minimizing the impact of uncertainty, i.e., noise affecting motor performance, can account for the choice of combined eye-head movements. This optimization criterion predicts all major features of natural eye-head movements-including the part where gaze is already on target and the eye counter-rotates - such as movement durations, relative eye-head contributions, velocity profiles, and the dependency of gaze shifts on initial eye position. As a critical test of this principle, we show that it also correctly predicts changes in eye and head movement imposed by an experimental increase in the head moment of inertia. This suggests that minimizing the impact of noise is a simple and powerful principle that explains the choice of a unique set of movement profiles and segment coordination in goal-directed action.

\section{Introduction}

Although a gaze shift can be realized by different combinations of eye-head movements, these movements are empirically found to be stereotyped in terms of relative eye and head contributions (Guitton and Volle, 1987; Freedman and Sparks, 1997), as well as velocity profiles of eye (Collewijn et al., 1988) and head (Zangemeister et al., 1981; Freedman and Sparks, 2000). The fact that the brain singles out one eye-head movement among many possibilities suggests an underlying optimization. Over the last decade, several optimization criteria have been proposed to explain the features of goal-directed movements (for review, see Todorov, 2004). Harris and Wolpert (2006) and Tanaka et al. (2006) tested the criteria of reaching the target as precisely and quickly as possible and show that, in contrast to other optimization criteria, this strategy accounts for the characteristics of natural eye movements. Timeaccuracy optimality can also be explained within the context of motor noise minimization. Harris and Wolpert (1998) already suggested that the neural control signal is contaminated by signal-dependent noise (SDN) with a standard deviation proportional to the control signal magnitude. van Beers $(2007,2008)$ proposed constant noise $(\mathrm{CN})$ as an additional source of uncertainty that varies independently of the motor signal. Minimizing the impact of SDN leads to accurate but long movements, because it

\footnotetext{
Received July 20, 2011; revised Aug. 31, 2011; accepted Sept. 21, 2011.

Author contributions: M.S., N.L., and S.G. designed research; M.S., N.L., and S.G. performed research; M.S., N.L., and S.G. contributed unpublished reagents/analytic tools; M.S., N.L., and S.G. analyzed data; M.S., N.L., and S.G. wrote the paper.

This work was supported by the German Federal Ministry of Education and Research Grants BMBF IFB 01 E00901 and BCCN 01GQ0440. We thank Sebastian Albrecht for his helpful advice and Judy Benson for copyediting the manuscript.

Correspondence should be addressed to Murat Sağlam, Institute for Clinical Neurosciences, Bernstein Center for Computational Neuroscience, Ludwig-Maximilians-University, Munich, Marchioninistrasse 23, 81377 Munich, Germany. E-mail: murat.saglam@|rz.uni-muenchen.de.

DOI:10.1523/JNEUROSCI.3721-11.2011

Copyright $\odot 2011$ the authors $\quad 0270-6474 / 11 / 3116185-09 \$ 15.00 / 0$
}

favors smaller control signals. However, long movement duration increases $\mathrm{CN}$-related variance, since $\mathrm{CN}$ increases with movement duration. Therefore, for a given movement amplitude, there is an optimal duration as an outcome of the compromise between the effect of SDN and CN (van Beers, 2008). This strategy accounts for the characteristics of single degree of freedom eye movements when the head is fixed. The complexity of neural control increases when several effectors work together as in combined eyehead movements. Addressing such combined movements, Kardamakis and Moschovakis (2009) proposed a minimum effort strategy. Although this model can account for eye-head movement characteristics, it has shortcomings: to account for stereotypical eyehead movements, the eye control signal has to be penalized for eccentricity. Moreover, the model only considers the first part of the gaze shift until the eye reaches maximum eccentricity. However, eye-head gaze shifts have two parts: one in which eye and head move together toward the target and one in which the head continues to move and gaze is stabilized by counter-rotation of the eye.

Here, we show that a simple optimal control model using parameters available to the CNS explains the entire eye-head movement, including the counter-rotation of the eye. The model minimizes the consequences of the sum of SDN and CN using the known plant dynamics of eye and head without additional constraints. It predicts the major kinematic features of eye-head movements such as relative contributions, initial position dependencies, movement durations, and stereotyped velocity profiles. We further show that the model not only accounts for these properties of natural eye-head movements, but also for the properties when the dynamics of the head are experimentally altered, i.e., with increased head inertia.

\section{Materials and Methods}

Subjects and experimental procedure. Ten healthy subjects (one woman and nine men; $39.7 \pm 6.3$ years of age) participated in the study. All gave their informed consent after explanation of the exper- 
imental procedure, which was approved by the ethics committee of the Medical Faculty of the Ludwig-Maximilians-University Munich and was in accordance with the Declaration of Helsinki. The data are published in Lehnen et al. (2009a), where the experimental procedure and data preprocessing are explained in detail. Angular positions of eye and head in space were recorded with the search-coil technique (Robinson, 1963). Coil and target signal data were sampled at a rate of $1 \mathrm{kHz}$ and calibration was performed as described previously (Glasauer et al., 2003). Subjects were instructed to perform gaze shifts in response to targets located horizontally $35^{\circ}$ or $40^{\circ}$ to the left or to the right of center. Eye-head movements in response to target jumps of $75^{\circ}$ and $80^{\circ}$ amplitude were analyzed. The target was flashed in darkness; it was visible for $<100 \mathrm{~ms}$ to prevent visual feedback. Subjects had to maintain gaze position in darkness until a new target appeared (randomly assigned time between 1.6 and $2.4 \mathrm{~s}$ ). After a control trial (natural condition, 43 target steps), the head moment of inertia was increased by eccentrically placed masses attached to a helmet (weighted condition, 43 target steps). This gave an additional moment of inertia of $0.0335 \mathrm{~kg} \cdot \mathrm{m}^{2}$ to the normal head moment of inertia, which was assumed to be $0.0148 \mathrm{~kg} \cdot \mathrm{m}^{2}$, as in Peng et al. (1996). This difference corresponds to a 3.3-fold increase. Before the experiments, subjects did not have any experience with increased head moment of inertia.

Model dynamics. The relationship between the net effector torque applied by the eye muscles and the horizontal eye movement dynamics is governed by the following second-order differential equation:

$$
\ddot{x}_{e}+\frac{\tau_{1}+\tau_{2}}{\tau_{1} \tau_{2}} \dot{x}_{e}+\frac{1}{\tau_{1} \tau_{2}} x_{e}=T_{e},
$$

where $x_{e}, \dot{x}_{e}$, and $\ddot{x}_{e}$ are position, velocity, and acceleration of the eye, respectively. The net torque applied to the eye $\left(T_{e}\right)$ is assumed to be a low-pass filtered version (with a time constant of $\tau_{e}$ ) of the control signal $\left(u_{e}\right)$ to account for the full muscle dynamics and the delay between neural activity and muscle response. Hence, the eye movement dynamics are governed by the following third-order plant:

$$
\dddot{x}_{e}+\frac{\tau_{1} \tau_{2}+\tau_{1} \tau_{e}+\tau_{2} \tau_{e}}{\tau_{1} \tau_{2} \tau_{e}} \ddot{x}_{e}+\frac{\tau_{1}+\tau_{2}+\tau_{e}}{\tau_{1} \tau_{2} \tau_{e}} \dot{x}_{e}+\frac{1}{\tau_{1} \tau_{2} \tau_{e}} x_{e}=\frac{1}{\tau_{e}} u_{e}
$$

When the eye state vector is defined as $\boldsymbol{x}_{e}=\left[\ddot{x}_{e}, \dot{x}_{e}, x_{e}\right]$, Equation 2 can be rewritten in the form of the state equation: $\dot{\boldsymbol{x}}_{e}=A_{e} \boldsymbol{x}_{e}+B_{e} u_{e}$, where $A_{e}$ and $B_{e}$ are as follows:

$$
\begin{gathered}
A_{e}=\left|\begin{array}{ccc}
-\frac{\tau_{1} \tau_{2}+\tau_{1} \tau_{e}+\tau_{2} \tau_{e}}{\tau_{1} \tau_{2} \tau_{e}} & -\frac{\tau_{1}+\tau_{2}+\tau_{e}}{\tau_{1} \tau_{2} \tau_{e}} & -\frac{1}{\tau_{1} \tau_{2} \tau_{e}} \\
1 & 0 & 0 \\
0 & 1 & 0
\end{array}\right|, \\
\\
\quad B_{e}=\left|\begin{array}{c}
\frac{1}{\tau_{e}} \\
0 \\
0
\end{array}\right| .
\end{gathered}
$$

The three parameters are chosen as $\tau_{1}=224, \tau_{2}=13$, and $\tau_{e}=10 \mathrm{~ms}$, as in Robinson et al. (1986), Harris and Wolpert (1998), Tanaka et al. (2006), and van Beers (2008).

Similarly, the relationship between the net effector torque applied to the head plant and the horizontal head movement dynamics are governed by the following second-order differential equation:

$$
I \ddot{x}_{h}+b \dot{x}_{h}+K x_{h}=T_{h}
$$

where $x_{\mathrm{h}}, \dot{x}_{\mathrm{h}}, \ddot{x}_{h}$ are position, velocity, and acceleration of the head, respectively. $I, b$, and $K$ stand for head moment of inertia, viscosity, and stiffness, respectively. The net torque applied to the head $\left(T_{h}\right)$ is estimated to be the low-pass filtered version (with a time constant of $\tau_{h}$ ) of the control signal $\left(u_{h}\right)$ and the head dynamics are governed by the following third-order plant:

$$
\dddot{x}_{h}+\left(\frac{1}{\tau_{h}}+\frac{b}{I}\right) \ddot{x}_{h}+\left(\frac{K}{I}+\frac{b}{\tau_{h} I}\right) \dot{x}_{h}+\frac{K}{\tau_{h} I} x_{h}=\frac{1}{\tau_{h} I} u_{h} .
$$

When head state vector is defined as $\boldsymbol{x}_{h}=\left[\ddot{x}_{h}, \dot{x}_{h}, x_{h}\right]$, Equation 5 can be written in the form of the state equation as $\dot{\boldsymbol{x}}_{h}=A_{h} \boldsymbol{x}_{h}+B_{h} u_{h}$, where $A_{h}$ and $B_{h}$ are as follows:

$$
A_{h}=\left|\begin{array}{ccc}
-\left(\frac{1}{\tau_{h}}+\frac{b}{I}\right) & -\left(\frac{K}{I}+\frac{b}{\tau_{h} I}\right) & -\frac{K}{\tau_{h} I} \\
1 & 0 & 0 \\
0 & 1 & 0
\end{array}\right|, B_{h}=\left|\begin{array}{c}
\frac{1}{\tau_{h} I} \\
0 \\
0
\end{array}\right| .
$$

The plant parameters are selected as $I=0.0148 \mathrm{~kg} \cdot \mathrm{m}^{2}, b=0.3 \mathrm{~N} \cdot \mathrm{m} \cdot \mathrm{s} /$ rad, $K=2.077 \mathrm{~N} \cdot \mathrm{m} / \mathrm{rad}$ (Peng et al., 1996), and $\tau_{h}=100 \mathrm{~ms}$ (modified from Tanaka et al., 2006). Instead of $0.1 \mathrm{~N} \cdot \mathrm{m} \cdot \mathrm{s} / \mathrm{rad}$ in Peng et al. (1996), $b$ is selected to be $0.3 \mathrm{~N} \cdot \mathrm{m} \cdot \mathrm{s} / \mathrm{rad}$, as in Tangorra et al. (2003); this fits the observed head velocity profile better. In our previous work (Lehnen et al., 2009a), we observed that the subjects found the optimal trajectories very quickly within the first trials under the weighted head moment of inertia condition. Our model predicts the situation when trajectories have already been optimized, so we used the updated value of head moment of inertia by setting $I$ to $0.0488 \mathrm{~kg} \cdot \mathrm{m}^{2}$, corresponding to a 3.3-fold increase. All other parameters remained unchanged. In the state space form, the gaze state vector $\left(\boldsymbol{x}_{g}\right)$ can be computed by the following equation:

$$
\begin{array}{r}
\boldsymbol{x}_{g}(t)=\boldsymbol{x}_{e}(t)+\boldsymbol{x}_{h}(t)=e^{A_{e} t} \boldsymbol{x}_{e}(0)+\int_{0}^{t} e^{A_{e}\left(t-t^{\prime}\right)} B_{e} u_{e}\left(t^{\prime}\right) d t^{\prime}+e^{A_{h} t} \boldsymbol{x}_{h}(0) \\
+\int_{0}^{t} e^{A_{h}\left(t-t^{\prime}\right)} B_{h} u_{h}\left(t^{\prime}\right) d t^{\prime} .
\end{array}
$$

Optimal control. We assume an open-loop strategy to minimize the sum of the consequences of SDN and CN. Gaze shifts can be divided into two parts: one in which eye and head move toward the target and one in which the head continues to move and the eyes counter-rotate. Eye movements during gaze shifts can thus be separated into two phases: (1) from the onset of the movement $\left(t_{0}\right)$ to the point where the eye reaches maximum eccentricity $\left(t_{e}\right)$, and (2) from $t_{e}$ to the point where eye and head stop $\left(t_{h}\right)$ (Fig. 1). Therefore, in the cost function (Eq. 8), SDN and $\mathrm{CN}$ costs of eye movements appear separately for the two phases as $J_{\mathrm{SDN}, e 1}, J_{\mathrm{SDN}, e 2}, J_{\mathrm{CN}, e 1}, J_{\mathrm{CN}, e 2}$; head movement costs are labeled as $J_{\mathrm{SDN}, h}$ and $J_{\mathrm{CN}, h}$.

$$
\begin{aligned}
J_{\text {Total }}=J_{\mathrm{SDN}, e 1}+J_{\mathrm{CN}, e 1}+J_{\mathrm{SDN}, e 2}+J_{\mathrm{CN}, e 2}+J_{\mathrm{SDN}, h}+J_{\mathrm{CN}, h} \\
=\int_{0}^{t_{e}} F_{\mathrm{SDN}, e 1}\left(t, u_{e}, \tau_{1,2, e}\right) d t+\int_{0}^{t_{e}} F_{\mathrm{CN}, e 1}\left(t, \tau_{1,2, e}\right) d t \\
+\int_{t_{e}}^{t_{h}} F_{\mathrm{SDN}, e 2}\left(t, u_{e}, \tau_{1,2, e}\right) d t+\int_{t_{e}}^{t_{h}} F_{\mathrm{CN}, e 2}\left(t, \tau_{1,2, e}\right) d t \\
+\int_{0}^{t_{h}} F_{\mathrm{SDN}, h}\left(t, u_{h}, I, b, K, \tau_{h}\right) d t+\int_{0}^{t_{h}} F_{\mathrm{CN}, h}\left(t, I, b, K, \tau_{h}\right) d t .
\end{aligned}
$$

In the total cost function (Eq. 8), $F_{\mathrm{SDN}, e}$ and $F_{\mathrm{SDN}, h}$ are the integrands of SDN costs, which are dependent on time, control signal, and the plant parameters of eye and head, respectively. Similarly, $F_{\mathrm{CN}, e}$ and $F_{\mathrm{CN}, h}$ account for the signal-independent noises in the system and are assumed to be dependent only on time and the plant parameters and independent of the control signals. 


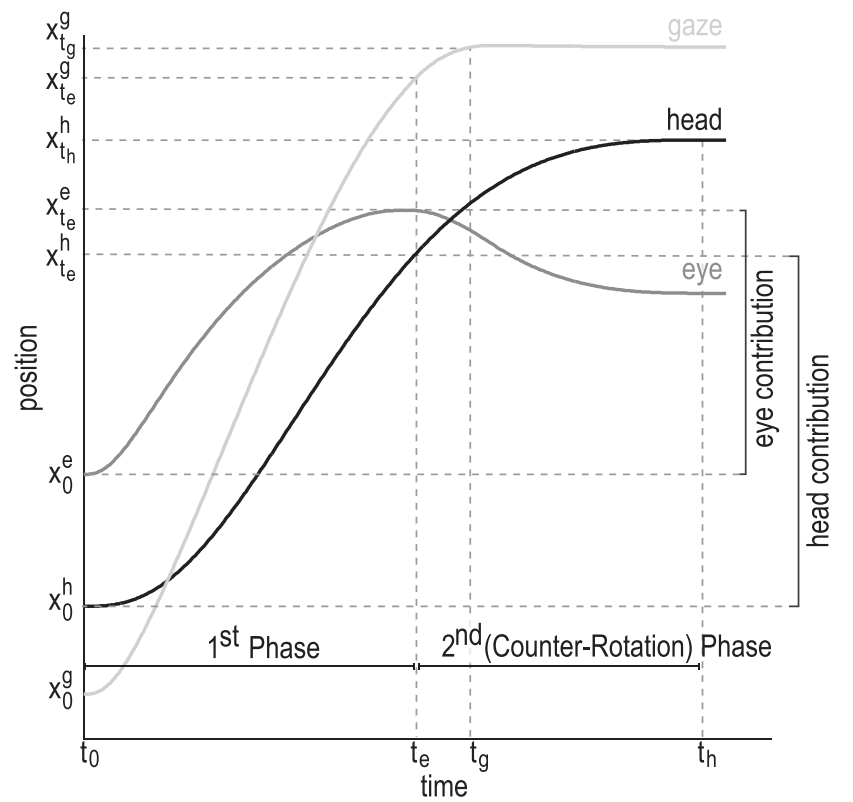

Figure 1. Eye-in-head (eye), head-in-space (head), and eye-in-space (gaze) position traces during an exemplary gaze shift following a horizontal target step. The gaze shift has two phases: (1) a movement of eye and head toward the target and (2) a phase where gaze is on target and the eyes counter-rotate while the head continues to move toward the target. The first phase lasts from the start $\left(t_{0}\right)$ to the point where the eyes reach maximum eccentricity $\left(t_{e}\right)$, the second from $t_{e}$ to maximum head eccentricity $\left(t_{h}\right)$. At $t_{e^{\prime}}$ the eyes start to counter-rotate. Gaze lands on the desired position at $t_{g} . x_{0}^{e}, x_{0}^{h}$, and $x_{0}^{g}$ are initial positions, $x_{t_{c}}^{e}, x_{t_{h}}^{h}$ and $x_{t_{g}}^{g}$ maximum eccentricities of eye, head, and gaze, respectively. Eye contribution is defined as $x_{t_{e}}^{e}-x_{0}^{e}$, head contribution is $x_{t_{e}}^{h}-x_{0}^{h}$

Signal-dependent noise. As was explained previously (Harris and Wolpert, 1998, 2006; van Beers, 2008), minimum variance minimizes SDNrelated variance during a postmovement period $\left(t_{p}\right)$ after the time when the target is reached $\left(t_{f}\right)$. In this study, $t_{f}$ can be either $t_{e}$ or $t_{h}$ for eye and head, respectively, and $t_{p}$ is fixed at $50 \mathrm{~ms}$, as in previous studies (Harris and Wolpert, 1998; van Beers, 2008). The standard deviation of SDN is assumed to be proportional to $u(t)$ with a weighting factor $(\alpha)$, so the variance at $t_{f}+t_{p}$ is derived as previously shown (Harris and Wolpert, 2006):

$$
\begin{aligned}
\operatorname{Var}\left[x\left(t_{f}+t_{p}\right)\right]=\int_{0}^{t_{f}+t_{p}} \alpha^{2} u^{2}(t) p^{2}\left(t_{f}+t_{p}-t\right) d t \\
\quad=\int_{0}^{t_{f}} \alpha^{2} u^{2}(t) p^{2}\left(t_{f}+t_{p}-t\right) d t+\int_{t_{f}}^{t_{p}} \alpha^{2} u^{2}(t) p^{2}\left(t_{f}+t_{p}-t\right) d t,
\end{aligned}
$$

where $p(t)$ is the impulse response function of the plant.

Since $u(t)$ during the postmovement period is assumed to be constant and equal to the elastic force of the plant multiplied by the eccentricity of the final position (Tanaka et al., 2006), the second term in Equation 9 becomes constant and can be excluded from the minimization process and from the cost function. The first term in Equation 9 provides a temporally nonuniform function to penalize the control signal for the period of $\left[0, t_{f}\right]$ to minimize the consequences of SDN. From Equation 9 the integrand of SDN cost in Equation 8 is as follows:

$$
F_{\mathrm{SDN}}[t, u, p(t)]=\alpha^{2} p^{2}\left(t_{f}+t_{p}-t\right) u^{2}(t) .
$$

For the head, $\alpha$ becomes $\alpha_{h}$ in Equation 10. As the eye control signal is determined in two phases, there are two constants for the eye $\left(\alpha_{e 1}\right.$ and $\left.\alpha_{e 2}\right)$. $p(t)$ stands for the impulse response function. Therefore, it is de- rived for eye $\left[p_{e}(t)\right]$ and head $\left[p_{h}(t)\right]$ plants separately, as shown by the following equations:

$$
\begin{gathered}
p_{e}(t)=\tau_{1} \tau_{2}\left(\frac{\tau_{1} e^{-\frac{t}{\tau_{1}}}}{\left(\tau_{1}-\tau_{2}\right)\left(\tau_{1}-\tau_{e}\right)}+\frac{\tau_{2} e^{-\frac{t}{\tau_{2}}}}{\left(\tau_{2}-\tau_{1}\right)\left(\tau_{2}-\tau_{e}\right)}+\frac{\tau_{e} e^{-\frac{t}{\tau_{e}}}}{\left(\tau_{e}-\tau_{1}\right)\left(\tau_{e}-\tau_{2}\right)}\right) \\
p_{h}(t)=c_{1}\left[e^{-\frac{t}{\tau_{h}}}-e^{-\frac{b t}{2 I}}\left[\cosh \left(\frac{t c_{2}}{I}\right)-\frac{c_{3} I}{c_{2}} \sinh \left(\frac{t c_{2}}{I}\right)\right]\right],
\end{gathered}
$$

where $c_{1}=\tau_{h} /\left(K \tau_{h}^{2}-b \tau_{h}+I\right), c_{2}=\sqrt{b^{2} / 4-K I}$, and $c_{3}=(2 I-$ $\left.b \tau_{h}\right) /\left(2 I \tau_{h}\right)$.

In general, for a single degree of freedom system, if movement time and initial and final states were fixed to $t_{f}, \boldsymbol{x}_{0}$, and $\boldsymbol{x}_{f}$, respectively, the optimization problem could be reduced to:

$$
\begin{gathered}
\min J_{\mathrm{SDN}}=\int_{0}^{t_{f}} \alpha^{2} p^{2}\left(t_{f}+t_{p}-t^{\prime}\right) u^{2}\left(t^{\prime}\right) d t^{\prime} \\
\text { subject to }: \boldsymbol{x}\left(t_{f}\right)=e^{A t_{f}} \boldsymbol{x}_{0}+\int_{0}^{t_{f}} e^{A\left(t_{f}-t^{\prime}\right)} B u\left(t^{\prime}\right) d t^{\prime}=\boldsymbol{x}_{f} .
\end{gathered}
$$

According to the Pontryagin's Minimum Principle (Kirk, 2004), the optimal control signal for a given $t_{f}$ with respect to the objective function is obtained by minimizing the following Hamiltonian function $\mathscr{H}$ with respect to $u(t)$ (van Beers, 2008):

$$
\mathscr{H}=\alpha^{2} p^{2}\left(t_{f}+t_{p}-t\right) u^{2}(t)+\Lambda^{T} e^{A\left(t_{f}-t\right)} B u(t),
$$

where $\Lambda=\left[\lambda_{1}, \lambda_{2}, \lambda_{3}\right]^{T}$ is a vector of three Lagrange multipliers. From the following equality, the control signal can be obtained by:

$$
\frac{\partial \mathscr{H}}{\partial u}=2 \alpha^{2} p^{2}\left(t_{f}+t_{p}-t\right) u(t)+\Lambda^{T} e^{A\left(t_{f}-t\right)} B=0
$$

$u(t)$ minimizes $\mathscr{H}$ if

$$
\frac{\partial^{2} \mathscr{H}}{\partial u^{2}}=2 \alpha^{2} p^{2}\left(t_{f}+t_{p}-t\right)>0 .
$$

Since $\alpha$ and $p(t)$ are positive, the condition of Equation 15 is satisfied and the optimal control signal $u^{*}(t)$ becomes

$$
u^{*}(t)=-\frac{\Lambda^{T} e^{A\left(t_{f}-t\right)} B}{2 \alpha^{2} p^{2}\left(t_{f}+t_{p}-t\right)} .
$$

By plugging $u^{*}(t)$ into the constraint equation in Equation 12, the Lagrange multiplier's vector $(\Lambda)$ can be determined for given $t_{f}, \boldsymbol{x}_{0}$, and $\boldsymbol{x}_{f}$. Thus, SDN cost of a single degree of freedom system can be computed as a function of movement duration and initial and final positions as $J_{\mathrm{SDN}}\left(t_{f}, \boldsymbol{x}_{0}, \boldsymbol{x}_{f}\right)$.

Constant noise. As explained in van Beers (2008), the inclusion of CN in the optimization problem given in Equation 12 would not change the solution (Eq. 16), since CN is independent of $u(t), \boldsymbol{x}_{0}$, and $\boldsymbol{x}_{\boldsymbol{f}}$. Therefore, $\mathrm{CN}$ cost was computed separately as a function of movement duration and plant dynamics. It was assumed that the standard deviation of $\mathrm{CN}$ is constant, thus the cost of $\mathrm{CN}$ is an increasing function of movement duration (Harris and Wolpert, 2006; van Beers, 2008). Then, the integrand of $\mathrm{CN}$ cost in Equation 8 is as follows:

$$
F_{\mathrm{CN}}=\beta^{2} p^{2}\left(t_{f}+t_{p}-t\right),
$$

where $\beta$ is the $\mathrm{CN}$ proportionality constant and appears as $\beta_{e 1}$ and $\beta_{e 2}$ for the first and second phases of the eye movement, respectively, and as $\beta_{h}$ for the head movement.

Simulations. Velocity and acceleration of eye and head were assumed to be zero at movement onset and end. Initial eye and head positions were set to $x_{0}^{e}=-10^{\circ}$ and $x_{0}^{h}=-25^{\circ}$ and desired final gaze position was selected as $x_{t_{g}}^{g}=35^{\circ}$ by referring to the experimental data. A hypothetical range of movement durations and final positions of eye and head was considered to compute $J_{\mathrm{SDN}, e 1}, J_{\mathrm{SDN}, e 2}, J_{\mathrm{CN}, e 1}, J_{\mathrm{CN}, e 2}, J_{\mathrm{SDN}, h}$, and $J_{\mathrm{CN}, h}$. 
Table 1. Experiment versus model predictions

\begin{tabular}{|c|c|c|c|c|c|}
\hline & \multicolumn{3}{|l|}{ Experimental data } & \multicolumn{2}{|c|}{ Simulated data } \\
\hline & Natural & Weighted & {$\left[F_{(1,9)}, p\right]$} & Natural & Weighted \\
\hline$t_{e}(\mathrm{~ms})$ & $217.83 \pm 7.11$ & $265.52 \pm 10.72$ & {$[35.1,<0.001]$} & 190 & 240 \\
\hline$t_{h}(\mathrm{~ms})$ & $460.47 \pm 20.12$ & $586.40 \pm 22.38$ & {$[73.0,<0.001]$} & 440 & 560 \\
\hline$v_{\max }^{e}(\% / s)$ & $244.15 \pm 12.18$ & $254.55 \pm 18.14$ & {$[1.05,0.331]$} & 294 & 300 \\
\hline$v_{\max }^{h}(\% / s)$ & $203.38 \pm 14.30$ & $142.83 \pm 10.44$ & {$[63.7,<0.001]$} & 253 & 188 \\
\hline$v_{\max }^{g}(\% / s)$ & $352.60 \pm 19.81$ & $304.57 \pm 15.36$ & {$[13.3,0.005]$} & 432 & 348 \\
\hline Eye contribution $\left({ }^{\circ}\right)$ & $30.30 \pm 1.99$ & $35.32 \pm 2.84$ & {$[14.7,0.004]$} & 32 & 36 \\
\hline Head contribution $\left({ }^{\circ}\right)$ & $27.71 \pm 1.50$ & $21.47 \pm 2.23$ & {$[32.0,<0.001]$} & 33 & 29 \\
\hline
\end{tabular}

Data is mean $\pm \mathrm{SE}(n=10) \cdot t_{e}$ and $t_{h}$ represent the durations until eye and head reach maximum eccentricity, respectively. $v_{\max }^{e}, v_{\max }^{h}$ and $v_{\max }^{g}$ show peak eye, head, and gaze velocities, respectively. The last two rows present eye and head contributions to the gaze shift.

First, the head movement duration was fixed at $t_{h}$ (Fig. 1) and $J_{\mathrm{SDN}, \mathrm{el}}$ was calculated for a hypothetical range of $t_{e}=\left[40, t_{h}\right] \mathrm{ms}$ and $x_{t_{e}}^{e}=$ $\left[-50^{\circ}, 50^{\circ}\right]$. With the $t_{h}$ fixed, we swept $x_{t_{h}}^{h}=\left[-120^{\circ}, 120^{\circ}\right]$ and computed the SDN cost of the head movement that satisfies $x_{t_{e}}^{e}+x_{t_{e}}^{h}=x_{t_{e}}^{g}$ and assigned the corresponding cost to $J_{\mathrm{SDN}, h}$. Apparently, at the point where the eye reaches maximum eccentricity and zero velocity $\left(t_{e}\right.$; Fig. 1$)$, the head continues to move. Ideally, the eye velocity should be instantaneously equal to the negative of the head velocity to stabilize gaze after $t_{e}$. In reality, it is not possible for the eyes to have such a prompt change in velocity. In other words, $t_{e}$ cannot be equal to $t_{g}$. Therefore, by inferring to our experimental data, the gap between $t_{e}$ and $t_{g}$ was set to be $60 \mathrm{~ms}$ (compare Fig. 1; it was experimentally found to be $60.7 \pm 7.3 \mathrm{~ms}$, mean \pm $\mathrm{SE})$. The offset between $x_{t_{e}}^{g}$ and $x_{t_{g}}^{g}$ was set to be $4^{\circ}$; thus the target was $x_{t_{e}}^{g}=$ $x_{t_{g}}^{g}-4^{\circ}$. This was consistent with the experimental data of $4.2 \pm 0.8^{\circ}$ (mean \pm SE). Varying the duration of the gap and the offset between $t_{e}$ and $t_{g}$ did not have a major impact on our simulations. To obtain the eye control signal during the counter-rotation phase $\left(\left[t_{e}, t_{h}\right]\right)$, the second phase of the eye control signal was numerically computed by minimizing the error between the desired gaze and the sum of eye and head positions for the period of $\left[t_{g}, t_{h}\right]$. The counter-rotation phase of the eye control signal was also analytically derived by plugging the desired eye state $\left(\boldsymbol{x}_{e}^{\prime}\right)$ into the following state equation:

$$
\dot{\boldsymbol{x}}_{e}^{\prime}=A_{e} \boldsymbol{x}_{e}^{\prime}+B_{e} u_{e} .
$$

$\boldsymbol{x}_{e}^{\prime}$ in Equation 18 is equal to $\left[-\ddot{x}_{h},-\dot{x}_{h}, \theta-x_{\mathrm{h}}\right]$, where $\theta$ is the desired gaze position. We confirmed that the numerically and analytically found eye control signals for the counter-rotation phase consistently matched. The SDN cost of the counter-rotation phase of the eye movement $\left(J_{\mathrm{SDN}, e 2}\right)$ was computed by plugging the computed control signal into Equation 10. $J_{\mathrm{CN}, e 1}$ was computed for the range of $t_{e}=\left[40, t_{h}\right] \mathrm{ms}$ and $J_{\mathrm{CN}, e 2}$ for the range of $t_{h}-t_{e}=\left[t_{h}-40,0\right] \mathrm{ms}$ to obtain a cost surface of $J_{\mathrm{SDN}, e 1}+J_{\mathrm{SDN}, e 2}+J_{\mathrm{SDN}, h}+J_{\mathrm{CN}, e 1}+J_{\mathrm{CN}, e 2}$. The minimum of that cost surface determines the optimal eye movement duration and optimal eye head contributions to the gaze shift. Finally, by repeating the process for different $t_{h}$, the minimum of $J_{\text {Total }}-J_{\mathrm{CN}, h}$ was determined as a function of $t_{h}$ and we included $J_{\mathrm{CN}, h}$ to determine the optimal $t_{h}$, which minimizes $J_{\text {Total }}$. The optimal duration of single degree of freedom movements does not depend on absolute values of weighting parameters (which are $\alpha$ and $\beta$ in this study) of SDN and CN but instead on their relative ratio (van Beers, 2008). This still holds for the present case, since the parameters $\alpha$ and $\beta$ appear multiplicatively in the individual terms of the overall cost $J_{\text {Total }}$ (Eq. 8). Accordingly, even though there are six noise terms, there are only five free parameters in the model.

As shown in Equation 12, $\alpha$ and the impulse response $p(t)$ act as penalty terms for the eye and the head control signals to be determined by the optimization process. Thus, the cost of SDN depends on the plant characteristics and on the weighting parameter $\alpha$. In the following, we set the SDN-related parameters $\alpha_{e 1}, \alpha_{e 2}$, and $\alpha_{h}$ to unity, so that the compromise between the impact of SDN of eye and head was purely determined by the eye and head plant dynamics. Hence, the CN-related parameters $\beta_{e 1}, \beta_{e 2}$, and $\beta_{h}$ were the only free parameters of the model and were determined to be $2.95,0.45$, and 0.72 , respectively, by fitting the simulated $t_{e}$ and $t_{h}$ to the ones experimentally observed under natural conditions (Table 1). Note that, after proper conversion and scaling, the ratio of $\beta_{e 1} / \alpha_{e 1}$ becomes $5.90 \cdot 10^{-5} \mathrm{~kg} \cdot \mathrm{m}^{2} / \mathrm{s}^{2}$, which is very close to the value of $7.96 \cdot 10^{-5} \mathrm{~kg} \cdot \mathrm{m}^{2} / \mathrm{s}^{2}$ reported by van Beers (2008). The free parameters remained unchanged for all further simulations.

\section{Results}

\section{Experimental results}

Eye-head movements were assessed under natural conditions and with the head moment of inertia increased (weighted condition). Gaze positions at the beginning and the end of the gaze shift were not significantly different under either condition (repeatedmeasures ANOVA, within factor: natural or weighted condition; compare Figs. $2 A$ and $3 A$ ). The initial gaze positions were $x_{0}^{g}=$ $-31.2 \pm 1.0^{\circ}$ (mean $\pm \mathrm{SE}$ ) for the natural condition and $x_{0}^{g}=$ $-31.4 \pm 1.6^{\circ}$ for the weighted condition $\left(F_{(1,9)}=0.081, p=0.783\right)$. End positions of gaze were $x_{t_{g}}^{g}=32.5 \pm 1.1^{\circ}$ for the natural condition and $x_{t_{g}}^{g}=31.5 \pm 1.6^{\circ}$ for the weighted condition $\left(F_{(1,9)}=\right.$ $0.839, p=0.384$ ). Similarly, repeated-measures ANOVA (within factor: natural or weighted condition) revealed no significant effect of increasing the head moment of inertia on initial eye $\left(x_{0}^{e}=\right.$ $-7.1 \pm 1.3^{\circ}$ for the natural condition, $x_{0}^{e}=-9.1 \pm 1.9^{\circ}$ for the weighted condition; $\left.F_{(1,9)}=4.548, p=0.062\right)$ and head positions $\left(x_{0}^{h}=-24.1 \pm 0.9^{\circ}\right.$ for the natural and $x_{0}^{h}=-22.4 \pm 1.4^{\circ}$ for the weighted condition; $F_{(1,9)}=15.62, p=0.068$ ). Figures $2 A$ and $3 A$ show mean eye, head, and gaze position (top) and velocity (bottom) traces from all subjects for natural and weighted conditions, respectively. Increasing the head moment of inertia significantly prolonged head movement duration and decreased peak head and gaze velocity (one-way repeatedmeasures ANOVA, significance level $p=0.05$; Table 1 ). In the weighted condition, the eyes took longer to reach the most eccentric position. Relative eye-head contributions were also significantly altered: in the weighted condition, head contribution was reduced and eye contribution increased. The gaze velocity profile was transformed from a symmetrical to a more skewed shape. Figures $2 B-D$ and $3 B-D$ show individual traces of eye, head, and gaze movements. Note that, although the mean eye velocity profile has a smooth transition from the first phase of the gaze shift $\left(\left[0, t_{e}\right]\right)$ to the counter-rotation phase $\left(\left[t_{e}, t_{h}\right]\right)$, individual traces of eye velocity show an abrupt change when counter-rotation begins (Figs. $2 B, 3 B$, arrow).

\section{Simulations}

Figure 4 demonstrates how the compromise between SDN and $\mathrm{CN}$ costs determines optimal eye-head movements. Computing SDN cost of the eye for different movement durations and contributions in the first phase of the eye movement produced the cost surface shown in Figure $4 A$. SDN cost is a decreasing function of movement duration $\left(t_{e}\right)$ and becomes higher for eccentric positions away from the center. The SDN cost for the 

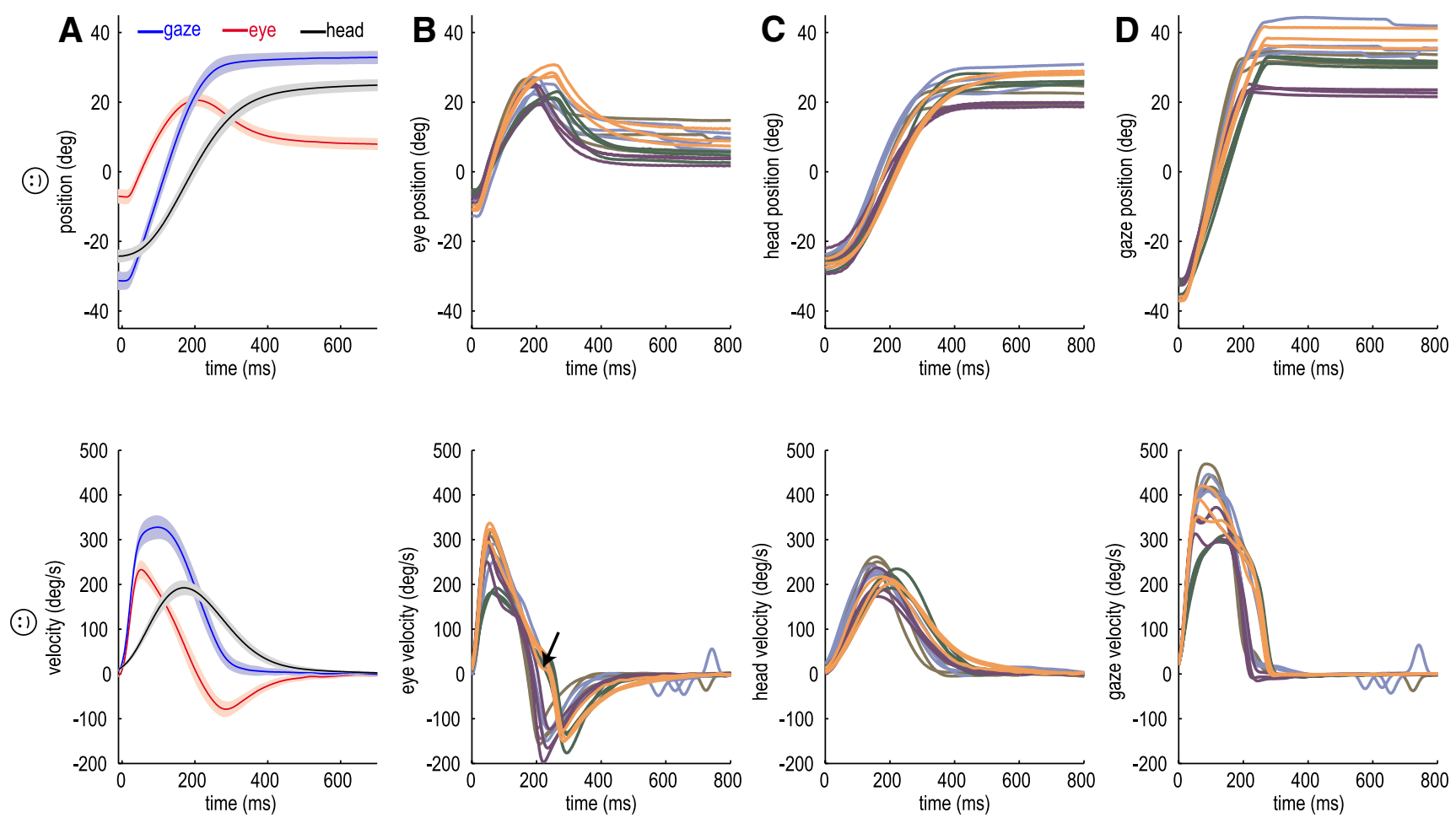

Figure 2. Position (top) and velocity (bottom) traces during gaze shifts in the unweighted, natural condition. $A$, Mean (solid line) and SEM (shaded area) traces of eye (red), head (black), and gaze (blue) from 10 subjects. Whereas head movement velocity profiles are symmetrically bell-shaped, eye-movement trajectories are skewed, with acceleration taking less time than deceleration. $\boldsymbol{B}-\boldsymbol{D}$, Individual traces of eye $(\boldsymbol{B})$, head $(\boldsymbol{C})$, and gaze $(\boldsymbol{D})$ from single subjects (each subject's trajectories are depicted with a different color). Note that the deceleration phase of the eye movement does not smoothly continue to the counter-rotation phase (arrow). This discontinuity can only be appreciated in the individual and not the average traces, due to the low-pass filtering effect of averaging.
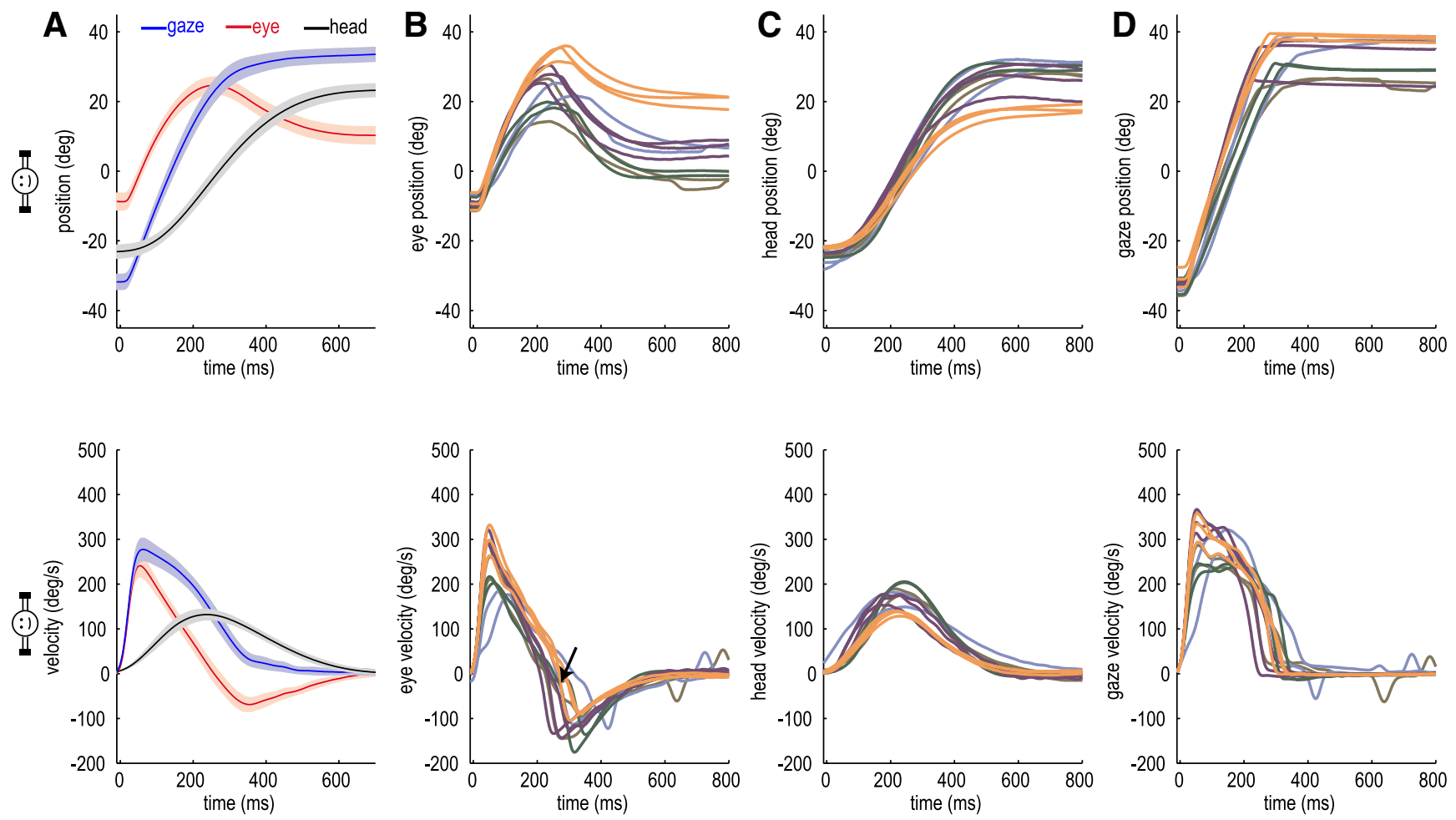

Figure 3. Position (top) and velocity (bottom) traces when the head moment of inertia is experimentally increased. $\boldsymbol{A}$, Mean traces (solid line) and SEM (shaded area) of eye (red), head (black), and gaze (blue) from the same 10 subjects as in Figure 2. Increasing the head moment of inertia leads to a decrease in peak head velocity and in head contribution and to an increase in head movement duration (compare to Fig. $2 A$ ). Note that the gaze velocity profile (bottom) is more skewed than in the natural condition (compare to Fig. $2 A$, bottom). Gaze positions at the beginning and the end of the gaze shift are not significantly different from the natural condition (Fig. $2 A$, top). Individual traces of eye (B), head (C), and gaze (D) from single subjects (colors for each subject as in Fig. 2). Note the discontinuity in the eye velocity trace between phase one and two of the gaze shift (arrow). 
A
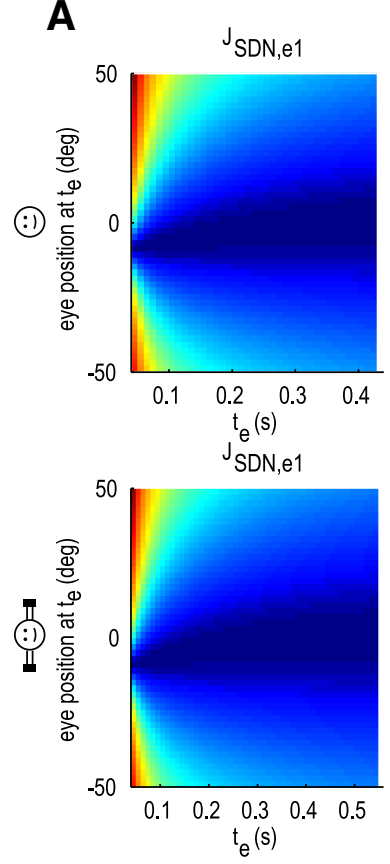

B
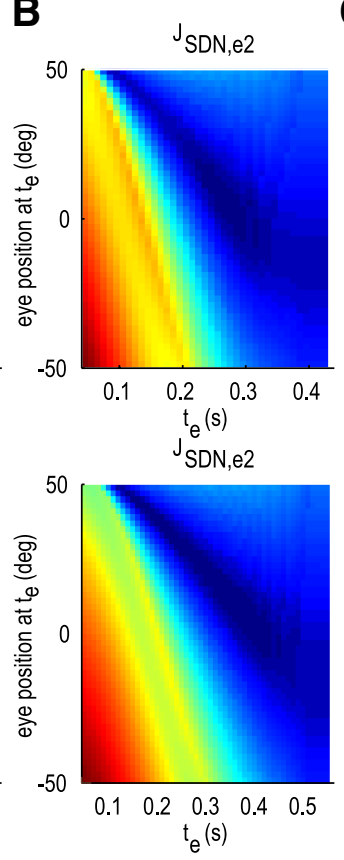

C
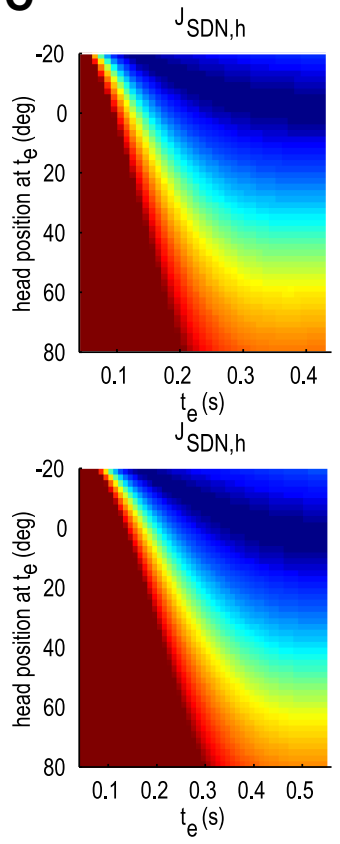

D
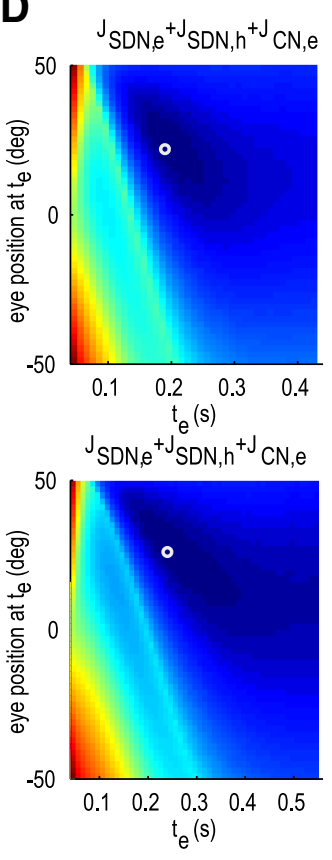

E
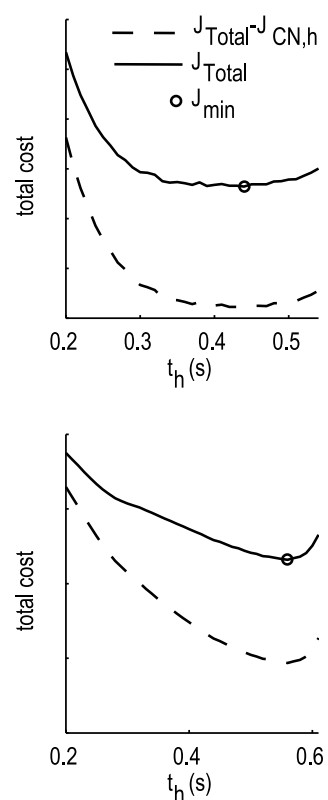

Figure 4. Simulated cost surfaces for a $70^{\circ}$ gaze shift in the natural condition (top) and with the head moment of inertia increased (bottom), illustrating how the compromise between SDN and CN costs determines optimal eye-head movements. Costs are color coded in log scale (red, high cost; blue, low cost) with the same ranges for the natural and the weighted conditions. $\boldsymbol{A}$, SDN cost of the eye $\left(J_{\mathrm{SDN}, e 1}\right)$ until it reaches maximum eccentricity (phase 1 ; defined in Fig. 1$)$ for different movement durations $\left(t_{e}\right)$ and contributions (eye position at $\left.t_{e}\right)$. SDN cost is a decreasing function of movement duration and gets higher for eccentric positions away from the center. The initial state of the eye is $x=\left[0,0,-10^{\circ}\right]$ and $x_{t_{e}}^{e}$ and $t_{e}$ are swept within the ranges of $\left[-50^{\circ}, 50^{\circ}\right]$ and $\left[0, t_{h}\right]$, respectively. $\boldsymbol{B}$, SDN cost of the eye during the counter-rotation phase $\left(J_{\text {SDN, }, 2}\right)$. SDN cost of the eye movement is both dependent on the duration and the amplitude of the counter-rotation of the eye. $C, J_{\text {SDN, },}$ for head movements from $x=\left[0,0,-25^{\circ}\right]$ to $x=\left[0,0, x_{t_{h}}^{h}\right]$, which satisfy $x_{t_{e}}^{e}+x_{t_{e}}^{h}=x_{t_{e}}^{g}$ for $x_{t_{e}}^{e}$ and $t_{e}$ within the ranges given in $\boldsymbol{A}$. The $y$-axis is inverted to better visualize the sum of $J_{\mathrm{SDN}, e}$ and $J_{\mathrm{SDN}, h} . D_{,} J_{\mathrm{SDN}, e 1}+J_{\mathrm{SDN}, e 2}+J_{\mathrm{SDN}, h}+J_{\mathrm{CN}, e 1}+J_{\mathrm{CN}, e 2}$. The gray circle indicates the global minimum of the surface which determines the optimal $t_{e}, x_{t_{e}}^{e}$ and $x_{t_{e}}^{h}$ for a given $t_{h}$. $\boldsymbol{E}$, Minimum of the surface costs given in $\boldsymbol{D}$ (dashed line) and of the total cost $\left(J_{\text {Total }}\right.$, solid line) versus $t_{h}$. The circle indicates the optimal $t_{h}$ for the global minimum of the total costs.

counter-rotation phase of the eye movement is dependent on both the duration of this phase $\left(t_{h}-t_{e}\right)$ and the amplitude of the counter-rotation of the eye (Fig. $4 B$ ). As shown in Figure $4 B$, SDN cost of the counter-rotation phase also favors longer $t_{e}$. The SDN cost of the head movement was computed so that the sum of eye-head contributions at different $t_{e}$ was equal to the desired gaze shift. The SDN cost of the head is also a decreasing function of $t_{e}$, because reaching a certain head contribution with a long movement duration, i.e., with a long $t_{e}$, requires a smaller control signal than a short duration. Figure $4 C$ shows this cost for the head position at $t_{e}$. Like eye SDN, the SDN cost of the head is higher if the head position is more eccentric (Fig. 4C). Therefore, for a given head movement duration and desired gaze target, the sum of SDN costs determines the optimal relative contributions of eye and head movements. This optimality depends on how the topology of the sum of SDN costs changes with respect to $t_{e}$. Unlike SDN costs, CN-related costs increase only with movement duration. Figure $4 D$ shows the sum of signal-dependent and constant noises $\left(J_{\mathrm{SDN}, e 1}+J_{\mathrm{SDN}, e 2}+J_{\mathrm{SDN}, h}+J_{\mathrm{CN}, e 1}+J_{\mathrm{CN}, e 2}\right)$ for a fixed head movement duration and desired gaze shift. Optimal $t_{e}$, together with optimal relative eye-head contributions, were determined by the global minimum of the surface (Fig. $4 D$, circle). Finally, the same procedure was repeated for different head movement durations and the optimal $t_{h}$ was determined. This gave the minimum total cost (Fig. $4 E$ ). The whole optimization process can be considered finding the global minimum in a total cost volume whose axes are $t_{e}, t_{h}$, and relative eye-head contribution for a desired gaze shift. Figure $4 A-D$ shows cost surfaces for optimal $t_{h}$ only. The optimal triplet of $t_{e}, t_{h}$, and relative eye-head contribution for natural and weighted conditions were used to compute optimal eye-head control signals from Equations 12 and 16 and the movement profiles (Fig. 5). Figure 5, top, shows that the model predicts eye-head movement kinematics, i.e., relative eye-head contributions (Fig. 5A) and eye, head, and gaze velocity profiles (Fig. $5 B$ ) in accordance with the experimental observations (natural condition; Fig. 2, Table 1).

For the weighted condition, the model has different predictions because increasing the head moment of inertia alters the impulse response function of the head $\left[p_{h}(t)\right.$, given in Eq. 11], which appears in the SDN-related cost term (Eq.12). Figure 4, D and $E$, bottom, demonstrates that optimal $t_{h}$ and $t_{e}$ are prolonged in the weighted condition. This is consistent with the experimental results (Fig. 3, Table 1). According to the model, peak velocity of the optimal head movement decreases when the head moment of inertia is increased (Fig. $5 B$, compare top and bottom); this closely resembles the experimental observations. Increasing the head moment of inertia affects eye-head contributions: eye contribution increases (Fig. 3, Table 1).

Since eye-head SDN costs are optimized by different temporally nonuniform functions (Eq. 11), optimal eye-head contributions are different for different gaze amplitudes. Figure 6, $A$ and $B$, shows simulated eye-head contributions for different gaze shift sizes. Eye movement contribution saturated for large gaze shifts, while head contribution increased with gaze shifts larger than $\sim 20^{\circ}$. This is in accordance with previous experimental observations (Fig. $6 A$, inset; $B$, inset, adapted from Guitton and Volle, 1987). The SDN cost surface was not symmetrical along the initial position of eye or head (Fig. $4 A, C$ ). In other words, for the 
A
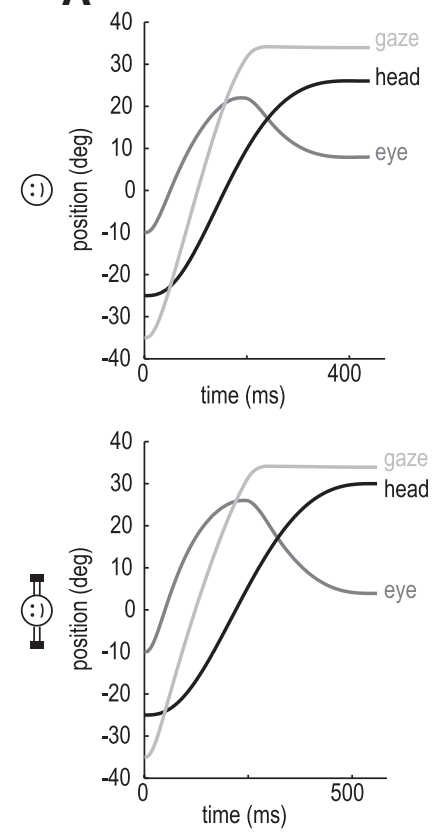

B
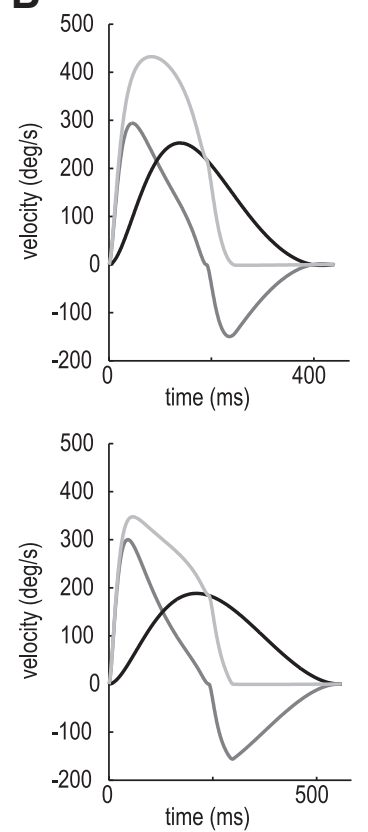

C
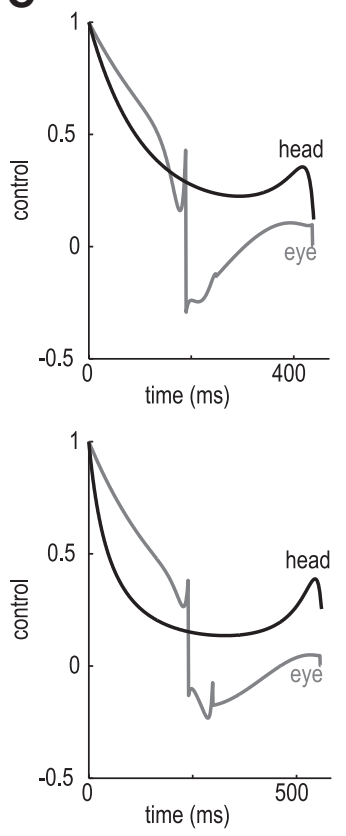

Figure 5. Simulation of eye and head movements with initial eye position at $-10^{\circ}$, head position at $-25^{\circ}$, and final gaze position at $35^{\circ}\left(70^{\circ}\right.$ gaze shift, velocity, and acceleration of eye and head are set to zero at the beginning and the end of the movement). Top, results in the natural condition; bottom, results for the weighted condition. $A$, Positions of eye (dark gray), head (black), and gaze (light gray). $\boldsymbol{B}$, Velocity profiles of eye (dark gray), head (black), and gaze (light gray). $\boldsymbol{C}$, Optimal control signals of eye ( $u_{e}$, dark gray) and head ( $u_{h}$, black). For illustration purposes, control signals are normalized.

model, it is cheaper to move the eye or head toward the central position rather than to eccentric directions. Therefore, the optimal solution is dependent on the initial eye and head positions. We simulated eye-head movements with different initial eye positions. Figure $6 C$ shows that eye contribution was high when the eye started from an eccentric position at the opposite side of the target. When the eye was initially on the same side as the target, eye contribution was smaller. This is consistent with experimental observations (Fig. 6C, inset, adapted from Freedman and Sparks, 1997). In this case, head contribution was consequently higher (Fig. 6D, compare to Fig. 6D, inset).

\section{Discussion}

In this study, we proposed an optimization criterion for the accuracy of gaze shifts, which can account for a variety of features observed during combined eye-head movements. This optimization criterion is based on a compromise between signal-dependent and signal-independent (constant) noise. We demonstrated that it is sufficient to take into account the dynamics of eye and head to capture stereotypical features of the entire head-free gaze shift, including the counter-rotation part, such as relative eye-head contributions, dependency of those contributions on initial eye position, and eyehead velocity profiles. Our model not only correctly predicts these features in the natural condition but also when head plant parameters are changed experimentally, i.e., when the head moment of inertia is increased.

\section{Optimization criteria for goal-directed movements}

Gaze shifts need to be accurate to be able to foveate the target. However, since noise jeopardizes accuracy, a useful strategy for gaze shifts is to minimize its effect. This consideration led to the minimum-variance model (Harris and Wolpert, 1998), which requires an additional criterion to define movement duration.
Instead of adding a new constraint, van Beers (2008) proposed extending the original model and determining the duration within the context of motor noise: penalizing signal-independent noise optimizes movement duration, as it accumulates with time; minimizing the impact of signal-dependent and -independent noise serves accuracy. This simple concept of minimizing the effect of signal-dependent and -independent noise can account for the stereotypical features of head-fixed eye movements (van Beers, 2008). Here, we investigated whether this concept holds for the complex situation of combined eye-head movements, which is further complicated by the fact that it consists of two phases: one in which eye and head move together toward the target and one in which gaze is stabilized on the target (the counter-rotation phase). Extending the idea of van Beers (2008) to penalize SDN- and CN-related consequences with weighting functions $\left[p_{e}(t)\right.$ and $\left.p_{h}(t)\right]$ times a constant ( $\alpha$ for SDN and $\beta$ for $\mathrm{CN}$ ), we have $\alpha_{e 1}, \alpha_{e 2}, \alpha_{h}, \beta_{e 1}$, $\beta_{e 2}$, and $\beta_{h}$ as weighting constants. The relative ratios of these parameters determine the optimal solution, yielding five free parameters. To fit the model, the free parameters were further reduced by setting $\alpha_{e 1}, \alpha_{e 2}$, and $\alpha_{h}$ to unity so that the penalty functions of SDN for eye and head are purely determined by the system dynamics rather than by additional weighting. The fact that our model is based on eye and head dynamics distinguishes it from that of Kardamakis and Moschovakis (2009). They proposed a minimum effort strategy, which penalizes the eye control signal with an eye-eccentricity-dependent quadratic function and the head control signal with a state-independent constant. It is widely accepted that the CNS has access to eye and head plant characteristics and that such internal models of plant dynamics are used, for example, in motor learning (Wolpert et al., 1995, 1998) or to generate an internal estimate of eye velocity (Glasauer, 2003). It appears more plausible that the motor system would use these eye-head dynamics rather than construct highly derived functions, such as a quadratic function of eye eccentricity, to estimate movement cost. The fact that our model depends on plant characteristics is further stressed by its correct prediction of the stereotypical changes of eye and head movements in response to an experimental change in plant characteristics, in our case, an increased head moment of inertia (Fig. 5A,B). There is a different control signal in the weighted as opposed to the natural condition (Fig. $5 C$ ). In contrast, a model not taking into account plant dynamics (Kardamakis and Moschovakis, 2009) would have an unchanged optimization criterion in the weighted condition and predict a motor command that leads to changes in the head movement via only the altered head dynamics. Such a model would be unable to predict the experimental findings in the weighted condition (Lehnen, 2006).

\section{Impact of feedback mechanisms}

We simulated the entire gaze shift, including the counterrotation phase, because it is critical for the optimal selection of the average behavior of eye and head. The consequence of disre- 

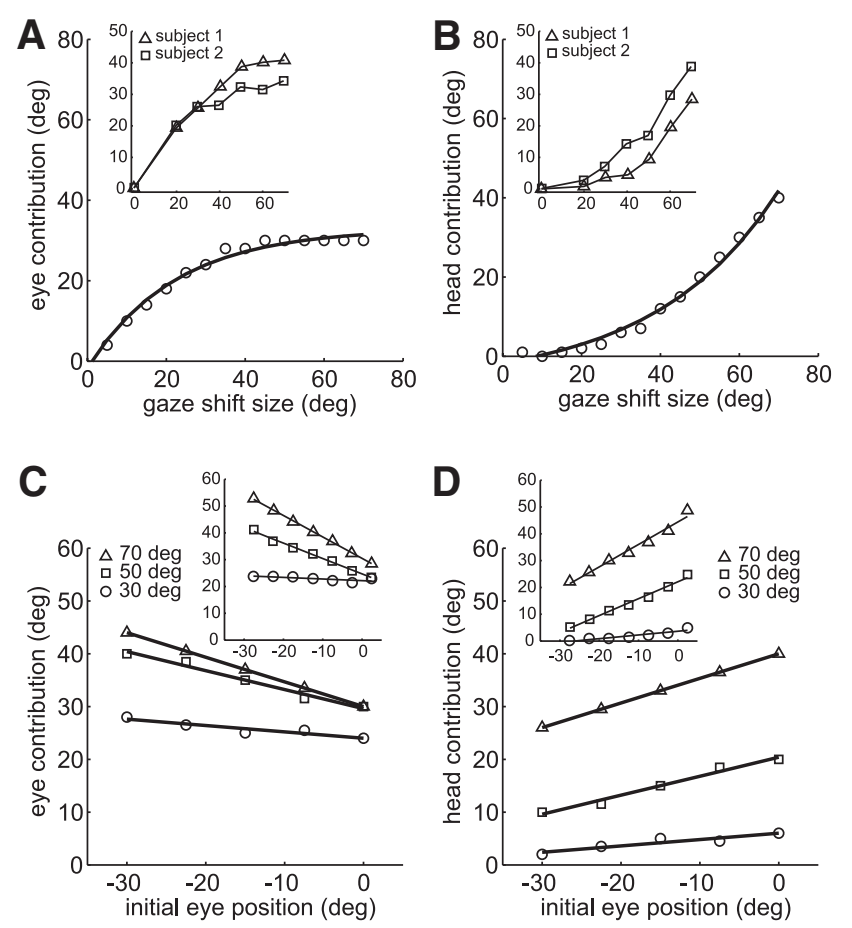

Figure 6. Main sequence and initial position dependency of simulated eye and head movements. $\boldsymbol{A}$, Eye contribution for different gaze shift sizes. $\boldsymbol{B}$, Head contribution for different gaze shifts. $\boldsymbol{A}, \boldsymbol{B}$, Initial eye and head positions are set to central positions. Insets are adapted from Guitton and Volle (1987) and show experimental results of human subjects. C, Eye contribution to gaze shifts of $30^{\circ}$ (circle), $50^{\circ}$ (square), and $70^{\circ}$ (triangle) with different initial eye positions. $D$, Head contribution to gaze shifts of $30^{\circ}$ (circle), $50^{\circ}$ (square), and $70^{\circ}$ (triangle) for different initial eye positions. $\boldsymbol{C}, \boldsymbol{D}$, Initial head position is set to the central position. Insets are adapted from Freedman and Sparks (1997) and show the dependency of eye and head contributions to different initial eye positions in rhesus monkeys.

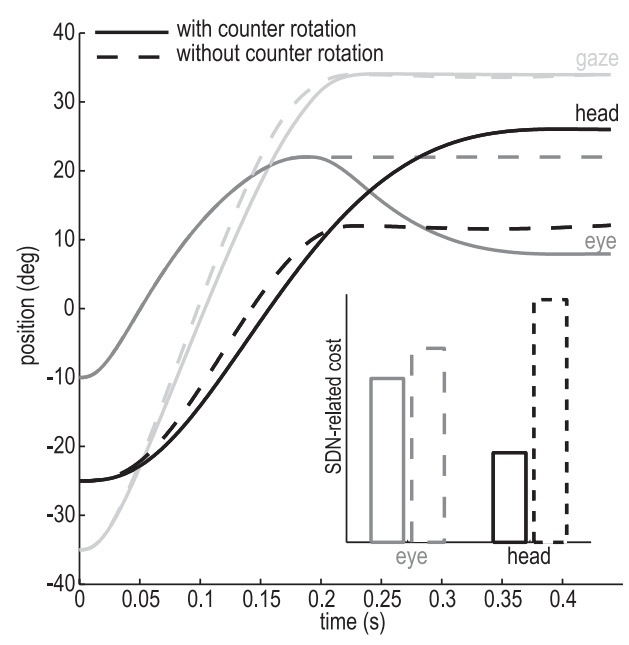

Figure 7. Eye, head, and gaze position traces for the simulated optimized movement (solid lines, with counter-rotation) and for a simulated hypothetical movement (dashed lines) producing a similar gaze shift but without counter-rotation of the eye. Inset shows eye and head SDN-related costs for the two strategies. For the eye and the head, SDN costs are higher during the hypothetical movement since larger control signals are required to keep the eye at an eccentric position and to stop the head at an earlier time. Note that the total elapsed time is equal for both strategies, thus $\mathrm{CN}$-related costs are identical.

garding this phase is demonstrated in Figure 7, which shows that the counter-rotation strategy of the present model is actually cost-efficient compared with a movement that is similar until the end of the gaze shift but lacks a counter-rotation afterward. The main advantage of a movement with counter-rotation is that head acceleration and deceleration can be lower, because the head movement does not have to finish together with the gaze movement, which in turn means that the head is less affected by SDN. Only for very low $\mathrm{CN}$ (for a gaze shift amplitude of $70^{\circ}, \beta_{e 1}<1.4$ ) would a strategy without counter-rotation be favored, which, however, would result in unnaturally long gaze-shift durations. During the counter-rotation phase, a short-latency and noisefree feedback mechanism based on the vestibulo-ocular reflex (VOR) could substantially correct for head movement-related errors in individual trials. However, the VOR latency precludes compensation of high-frequency noise due to increasing phase lag; the VOR gain is close to unity only at a limited frequency range (Tabak and Collewijn, 1994), and it has been shown to be attenuated during active head movements (Tabak et al., 1996). Furthermore, the VOR itself is also not noise-free (Sadeghi et al., 2007). Patients lacking a VOR due to peripheral vestibular damage still show counter-rotation of the eyes during active gaze shifts (Maurer et al., 1998; Lehnen et al., 2009b). Due to these facts, we assume that the possible feedback about head velocity provided by the vestibulo-ocular reflex is not taken into account by the optimization strategy. Rather, the optimal movement is chosen according to a strategy that does not rely on VOR correction but rather takes the head-related costs throughout the whole movement into account for the optimization process.

Like many previous optimization models (Uno et al., 1989; Harris and Wolpert, 1998, 2006; Tanaka et al., 2006; van Beers, 2008; Kardamakis and Moschovakis, 2009), our model only considers feedforward mechanisms. This is appropriate to reflect well practiced movements, but obviously, feedback mechanisms involved in the control of head movements can play a role during active gaze shifts, e.g., by correcting undesired deviations on-line and from trial-to-trial. Nonetheless, for unperturbed gaze shifts, based on previous work (Todorov and Jordan, 2002; Pham and Hicheur, 2009), it is reasonable to assume that an optimal feedback control would not alter the general system behavior derived here, but would only yield a smaller variance, i.e., increase accuracy even more.

\section{Contributions of eye and head constant noise}

Our model has only three free parameters $\left(\beta_{e 1}, \beta_{e 2}\right.$, and $\left.\beta_{h}\right)$; they determine the relative weight of $\mathrm{CN}$ versus SDN costs. These parameters were chosen to fit the experimentally observed eyehead movement durations under natural conditions. The same parameters were used to simulate the situation when the head moment of inertia was increased. The weighting factor for the first phase of the eye movement $\left(\beta_{e 1}=2.95\right)$ markedly exceeded the factors for the counter-rotation phase of the eye movement $\left(\beta_{e 2}=0.45\right)$ and the one for the head movement $\left(\beta_{h}=0.72\right)$. This implies that the consequences of $\mathrm{CN}$ during the first phase of the eye movement are more critical to the system than those during the counter-rotation phase and of those of the head movement. The difference in $\beta_{e 1}$ and $\beta_{e 2}$ could also be related to different neural origins of the control signals during the first and the second phases of the eye movements. In contrast, $\beta_{e 2}$ and $\beta_{h}$ are of similar magnitude, indicating that the eye control signal depends on the head control signal during the counter-rotation phase (Eq. 18).

\section{Comparison of simulations and experimental data}

Our model correctly predicts eye-head contribution, initial position dependency, and characteristic velocity profiles. The model also predicts increased movement duration under the 
condition of increased head moment of inertia. Since the plant characteristics mainly determine optimal eye and head contributions, the model correctly predicts relative eye contribution with increased head moment of inertia. Similarly, initial positiondependency is determined on the basis of plant characteristics: Figure 4, $A$ and $C$, shows that eye-head SDN cost surfaces are not symmetrical along the initial position axis. It is more costly, for example, to move the eye or head outward than inward because of the elastic force pulling them to the center; SDN cost function favors central positions for eye and head (Fig. 4). In contrast to the minimum-effort approach (Kardamakis and Moschovakis, 2009), which explicitly penalizes eye eccentricity with an additional penalty function, in our model the initial position dependence is a consequence of the plant dynamics. In accordance with Harris and Wolpert (1998) and Tanaka et al. (2004), our model also accounts for the skewed eye and bell-shaped head velocity profiles (Fig. 5). Since the relative latencies and amplitudes of peak eye and head velocities depend on the relation of the plant dynamics of the eye and the head, the skew of the gaze velocity profile is more pronounced with increased head moment of inertia. This phenomenon was observed both in the experiments (Figs. 2A, 3A) and in the simulations (Fig. 5).

\section{References}

Collewijn H, Erkelens CJ, Steinman RM (1988) Binocular coordination of human horizontal saccadic eye-movements. J Physiol 404:157-182.

Freedman EG, Sparks DL (1997) Eye-head coordination during headunrestrained gaze shifts in rhesus monkeys. J Neurophysiol 77:2328-2348.

Freedman EG, Sparks DL (2000) Coordination of the eyes and head: movement kinematics. Exp Brain Res 131:22-32.

Glasauer S (2003) Cerebellar contribution to saccades and gaze holding: a modeling approach. Ann N Y Acad Sci 1004:206-219.

Glasauer S, Hoshi M, Kempermann U, Eggert T, Büttner U (2003) Threedimensional eye position and slow phase velocity in humans with downbeat nystagmus. J Neurophysiol 89:338-354.

Guitton D, Volle M (1987) Gaze control in humans: eye-head coordination during orienting movements to targets within and beyond the oculomotor range. J Neurophysiol 58:427-459.

Harris CM, Wolpert DM (1998) Signal-dependent noise determines motor planning. Nature 394:780-784

Harris CM, Wolpert DM (2006) The main sequence of saccades optimizes speed-accuracy trade-off. Biol Cybern 95:21-29.

Kardamakis AA, Moschovakis AK (2009) Optimal control of gaze shifts. J Neurosci 29:7723-7730.

Kirk DE (2004) Optimal control theory: an introduction. Mineola, NY: Dover.

Lehnen N (2006) The effect of increased head inertia on eye-head control in human gaze shifts: analysis and mathematical modeling of a motor system. Thesis. Faculty of Medicine, Ludwig-Maximilians-University Munich.

Lehnen N, Büttner U, Glasauer S (2009a) Vestibular guidance of active head movements. Exp Brain Res 194:495-503.

Lehnen N, Büttner U, Glasauer S (2009b) Head-free gaze control in humans with chronic loss of vestibular function. Ann NY Acad Sci 1164:409-412.

Maurer C, Mergner T, Becker W, Jürgens R (1998) Eye-head coordination in labyrinthine-defective humans. Exp Brain Res 122:260-274.

Peng GC, Hain TC, Peterson BW (1996) A dynamical model for reflex activated head movements in the horizontal plane. Biol Cybern 75:309-319.

Pham QC, Hicheur H (2009) On the open-loop and feedback process that underlie the formation of trajectories during visual and nonvisual locomotion in humans. J Neurophysiol 102:2800-2815.

Robinson DA (1963) A method of measuring eye movement using a scleral search coil in a magnetic field. IEEE Trans Biomed Eng 10:137-145.

Robinson DA, Gordon JL, Gordon SE (1986) A model of the smooth pursuit eye movement system. Biol Cybern 55:43-57.

Sadeghi SG, Chacron MJ, Taylor MC, Cullen KE (2007) Neural variability, detection thresholds, and information transmission in the vestibular system. J Neurosci 27:771-781.

Tabak S, Collewijn H (1994) Human vestibuloocular responses to rapid, helmet-driven head movements. Exp Brain Res 102:367-378.

Tabak S, Smeets JB, Collewijn H (1996) Modulation of the human vestibuloocular reflex during saccades: probing by high-frequency oscillation and torque pulses of the head. J Neurophysiol 76:3249-3263.

Tanaka H, Tai M, Qian N (2004) Different predictions by the minimum variance and the minimum torque-change models on the skewness of movement velocity profiles. Neural Comput 16:2021-2040.

Tanaka H, Krakauer JW, Qian N (2006) An optimization principle for determining movement duration. J Neurophysiol 95:3875-3886.

Tangorra JL, Jones LA, Hunter IW (2003) Dynamics of the human headneck system in the horizontal plane: joint properties with respect to a static torque. Ann Biomed Eng 31:606-620.

Todorov E (2004) Optimality principles in sensorimotor control. Nat Neurosci 7:907-915.

Todorov E, Jordan MI (2002) Optimal feedback control as a theory of motor coordination. Nat Neurosci 5:1226-1235.

Uno Y, Kawato M, Suzuki R (1989) Formation and control of optimal trajectory in human multijoint arm movement: minimum torque-change model. Biol Cybern 61:89-101.

van Beers RJ (2007) The sources of variability in saccadic eye movements. J Neurosci 27:8757-8770.

van Beers RJ (2008) Saccadic eye movements minimize the consequences of motor noise. PLoS One 3:e2070.

Wolpert DM, Ghahramani Z, Jordan MI (1995) An internal model for sensorimotor integration. Science 269:1880-1882.

Wolpert DM, Miall RC, Kawato M (1998) Internal models in the cerebellum. Trends Cogn Sci 2:338-347.

Zangemeister WH, Jones A, Stark L (1981) Dynamics of head movement trajectories: main sequence relationship. Exp Neurol 71:76-91. 